\title{
Beam Steering at Higher Photonic Bands and Design of a Directional Cloak Formed by Photonic Crystals
}

\author{
N. Yogesh and V. Subramanian \\ Microwave Laboratory, Department of Physics, Indian Institute of Technology Madras, Chennai-600036, INDIA. \\ *corresponding author, E-mail: manianvs@iitm . ac . in
}

\begin{abstract}
Beam s teering $d$ ue $t$ o a nomalous di spersion a thi gher photonic bands in dielectric photonic crystal is reported in this $\mathrm{w}$ ork. $\mathrm{B}$ ased on $\mathrm{t}$ his $\mathrm{c}$ oncept, directional $\mathrm{c}$ loak i $\mathrm{s}$ designed $\mathrm{t}$ hat co nceals a larger dimensional s cattering object ag ainst $\mathrm{t}$ he normal $\mathrm{i}$ ncident, 1 inearly $\mathrm{p}$ olarized electromagnetic waves.
\end{abstract}

\section{Introduction}

Photonic c rystals ( PCs) are the p eriodic arrangement of dielectric/magnetic $\mathrm{c}$ onstituents $\mathrm{i} \mathrm{n}$ one, $\mathrm{t}$ wo, $\mathrm{t}$ hree dimensions o ffer $\mathrm{v}$ arieties of electromagnetic phe nomena such as the band gap, ne gative refraction, self-collimation, ultra-divergence, s ub-wave focusing a nd s o o n [1]. T heir utilities in m icrowave photonics, communications systems and optical sciences are on high demand. Particularly, these periodic structures serve as an avenue to realize some of the transformational el ectromagnetic ap plications s uch as the cloaking/invisibility [2, $3-5]$ and source $t$ ransformation devices [6].

Comparing $\mathrm{t} \mathrm{o}$ the metamaterials ( sub-wavelength structures that $\mathrm{p}$ ossess the negative di spersion characteristics), $\mathrm{P}$ Cs neither possess $\mathrm{t}$ he ne gative permittivity $n$ or $t$ he negative $p$ ermeability. $H$ owever, $t$ he anomalous di spersion in periodic $\mathrm{s}$ tructures e ntails the realization o f t ransformational applications. For i nstance, conformal mapping a pproach is us ed for the design of a cloaking de vice using $m$ etamaterials [7-11] but in P Cs, negative $\mathrm{r}$ efraction [3], gradient i ndex a pproach [4], an d waveguiding $\mathrm{m}$ echanisms [5] are envisaged $\mathrm{f}$ or the realization of a cloaking structure. Similarly, the reciprocal transformation is used for the design of a source transformer using $\mathrm{m}$ etamaterials [12] but $\mathrm{PC}$ simply $\mathrm{r}$ ealizes $\mathrm{i}$ ts functionality e ntirely from a d ielectric $\mathrm{s}$ tructure based o $\mathrm{n}$ the near-band gap phenomena [6].

In a ddition to the a bove a pproaches, this $p$ aper $r$ eports the beam steering mechanism at higher bands in PCs at the vicinity of the strong $\mathrm{d}$ ielectric a nisotropy. Ba sed on this concept, a directional cloaking s tructure is de signed for a normal incident, linearly polarized e-m wave. The proposed design is s calable at a $11 \mathrm{l}$ ength-scales $r$ anging from ra dio frequencies to $v$ isible 1 ight and it could be $u$ seful for the development of concealment a nd s tealth $t$ echnology i $n$ communication systems.

\section{Beam Steering at Higher Photonic Bands}

It is possible to steer the e-m w ave as shown in Fig. 1(a) that $\mathrm{s}$ hows $t$ he $\mathrm{E}_{\mathrm{z}}$ (Transverse $\mathrm{M}$ agnetic ( $\mathrm{T} \mathrm{M}$ ) $\mathrm{m}$ ode polarization) field map at $26.77 \mathrm{GHz}$ for a photonic prism made o f a $\mathrm{s}$ quare 1 attice ar rangement o f g lass rods o $\mathrm{f}$ relative dielectric permittivity of $5.5 \mathrm{w}$ ith the radius of 0.3 $\mathrm{cm}$ in air background. Lattice constant of the PC is $0.8 \mathrm{~cm}$. This field computation is performed with the finite-element methodology based e-m solver COMSOL RF Module [13]. It is observed that the normal incident e-m beam undergoes positive be am steering at $t$ he output i nterface of a $\mathrm{P} \mathrm{C}$ wedge.

It is important to note that this kind of beam steering is not $r$ ealizable i $n$ an o ptically denser medium as the $\mathrm{e}-\mathrm{m}$ beam is internally reflected when it passes from the denser to the rarer medium. This suggests that the prism wedge's effective i ndex i s 1 ess $t$ han $t$ he ai $r b$ ackground an $d t$ he steering direction indicates the positive index. While it is intricate $t$ of ind a naturally a vailable $m$ aterial $w$ ith $t$ he positive $\mathrm{i}$ ndex $\mathrm{l}$ ess $\mathrm{t}$ han $\mathrm{t}$ he a ir, $\mathrm{P}$ Cs a re $\mathrm{t}$ he $\mathrm{s}$ implest artificial composites to show this kind of dispersion behavior.

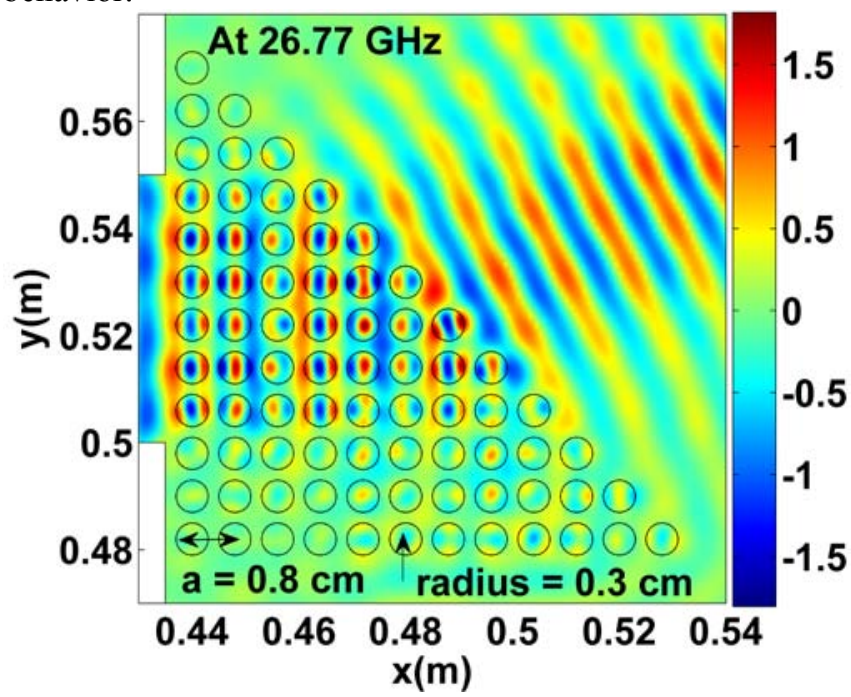

Figure 1: $E_{z}$ field map at $26.77 \mathrm{GHz}$ for a normal incident e-m wave on a photonic glass prism.

To gain further understanding about its mechanism, band structure and ray tracing results are obtained using the plane 
wave methodology based free e-m solver MPB [14]. Figure 2(a) shows the TM mode band structure of the square lattice glass PC with the normalized radius of $0.375 a$, where $a$ is the lattice constant. It is found that the steering frequency $26.77 \mathrm{GHz}$ (normalized angular frequency is $0.714(2 \pi \mathrm{c} / a)$ ) overlaps $w$ ith the fifth and s ixth ba nds of the TM mode band structure in Figure 2(a). It is observed that this steering behaviour is a characteristic feature of the higher photonic bands.
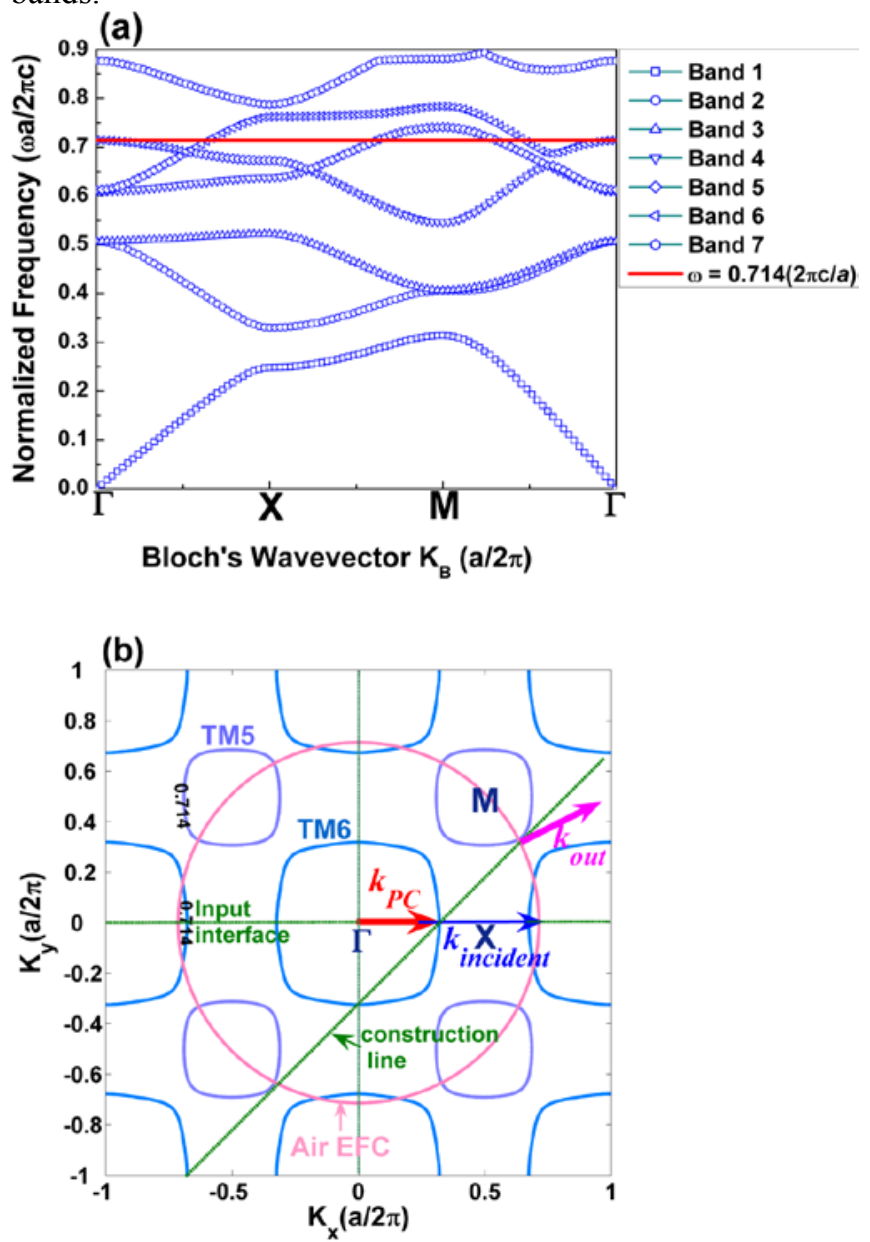

Figure 2: (a) TM mode b and s tructure of a s quare lattice glass PC. Operating frequency $0.714(2 \pi \mathrm{c} / a)$ i s i ndicated with $\mathrm{t}$ he $\mathrm{s}$ olid $\mathrm{h}$ orizontal $\mathrm{l}$ ine in the plot. (a) $\mathrm{W}$ avevector diagram i $\mathrm{n} t$ he $\mathrm{r}$ epeated $\mathrm{B}$ rillouin $\mathrm{z}$ one a $\mathrm{t} 0.714(2 \pi \mathrm{c} / a)$. $k_{\text {incident }}, k_{P C}$ and $k_{\text {out }}$ indicate $\mathrm{t}$ he di rections of $\mathrm{i}$ ncident, propagated and refracted components respectively. $\Gamma, X$ and $\mathrm{M}$ are the highest symmetry points of the Brillouin zone of the square lattice.

To e xpedite $\mathrm{t}$ his, one $\mathrm{m}$ ay $\mathrm{l}$ ook a round $\mathrm{t}$ he nature of dispersion a $t$ va rious bands. I $n$ ge neral, e ffective homogenization is possible at first and second band of the PC. However, the prism we dge d oes $n$ ot show the beam steering at first band due to the higher refractive index than the air $b$ ackground. $S$ econd $b$ and $f$ requencies are well known for negative dispersion and one can steer the beam negatively. It may be noticed that $\mathrm{V}$ anbésien et al [3] had designed $\mathrm{a}$ directional $\mathrm{cl}$ oak $\mathrm{b}$ ased $\mathrm{o} \mathrm{nt}$ he $\mathrm{n}$ egative dispersion at second band frequencies. Third and forth band of PC reveal an extreme anisotropy towards the $\Gamma$ symmetry point of the square lattice so that the e-m beam will largely disperse upon the normal incidence. F ifth and s ixth bands are the regimes of special interest but it is noticed that the fifth band has a partial band gap towards the $\Gamma \mathrm{X}$ symmetry direction. For instance, the red s olid line (at $0.714(2 \pi \mathrm{c} / a)$ ) drawn i n t he ba nd s tructure (Figure 2 (a)) i ndicates t he opening of band gap along $\Gamma \mathrm{X}$ symmetry direction for the fifth band so that the fifth band forbids the normal incident light to enter into the prism. On the other hand, sixth band dispersion at the same frequency al lows the beam to travel along the $\Gamma X$ symmetry direction of the square lattice PC. Hence the beam s teering mechanism i s f ocused on the dispersion nature around the sixth band frequencies.

The wavevector diagram gi ven i $\mathrm{F}$ igure $2(\mathrm{~b}) \mathrm{a} \mathrm{t} \mathrm{t}$ he steering frequency $0.714(2 \pi \mathrm{c} / a)$ clarifies the observed beam steering. S ince this frequency is shared by fifth a nd sixth bands, two d ifferent di spersion contours are wi tnessed around $\Gamma$ and $M$ symmetry directions in F igure 2(b). T he corresponding air dispersion contour at $0.714(2 \pi \mathrm{c} / a)$ includes $\mathrm{t}$ he $\mathrm{PC} \mathrm{c}$ ontours and thus i ndicates $\mathrm{t}$ he $\mathrm{l}$ ower effective index of the PC.

When a normal i ncident e-m b eam (represented b y $k_{\text {incident }}$ in Figure 2(b)) is excited towards the $\Gamma \mathrm{X}$ symmetry direction, e-m ray is maintaining its direction inside the $\mathrm{PC}$ with a minimal di vergence owing to the curvature of the TM6 contour (refer $k_{P C}$ in Figure 2(b)). The refraction at the second i nterface ( slanted s urface of the $\mathrm{P} \mathrm{CW}$ edge) is determined by the continuity e quation, which reveals the positive beam steering of the e $-\mathrm{m}$ beam in the a ir medium (refer $k_{\text {out }}$ in Figure 2(b)).

This steering property could be employed for the design of be am e xpanders a nd c ompressors based on the $\mathrm{p}$ rism combinations. In this work, the steering property is used for the design of a directional cloak.

\section{Realization of a Directional Cloak}

Directional cl oak c onceals the s cattering o bjects i $\mathrm{n}$ one direction at 1 east for a normal i ncident, 1 inearly $p$ olarized e-m waves. It has a pplications in the development of light protection c ircuits $\mathrm{i} \mathrm{nm}$ icrowave photonics a nd stealth mechanism in communication s ystems. This pa per reports the de sign o f a one such ge ometry based on the aforementioned beam steering concept. Figure 3 shows the layout of a directional cloak.

It c onsists of four P C prisms, where the 1 eft a nd right wedges are separated by a distance of $2 d_{1}$, where $d_{1}$ is fixed to $6.1 \mathrm{~cm}$. Similarly, top and bottom w edges a re separated by a distance of $2 \mathrm{~d}_{2}$, w here $\mathrm{d}_{2}$ is fixed to $2.1 \mathrm{~cm}$. I t is expected that the combination of right angled wedges would split the be am into two c omponents. T op and bot tom wedges a re used $t$ o guide $t$ he $\mathrm{e}-\mathrm{m}$ be am a round $\mathrm{t}$ he scattering object (perfect el ectric conductor-PEC) placed at the centre of the configuration. The prism at the output port reconstructs the separated beams into a single beam. At the centre of the configuration, a PEC object of diameter of 2 $\mathrm{cm}$ is placed for the concealment purpose. 


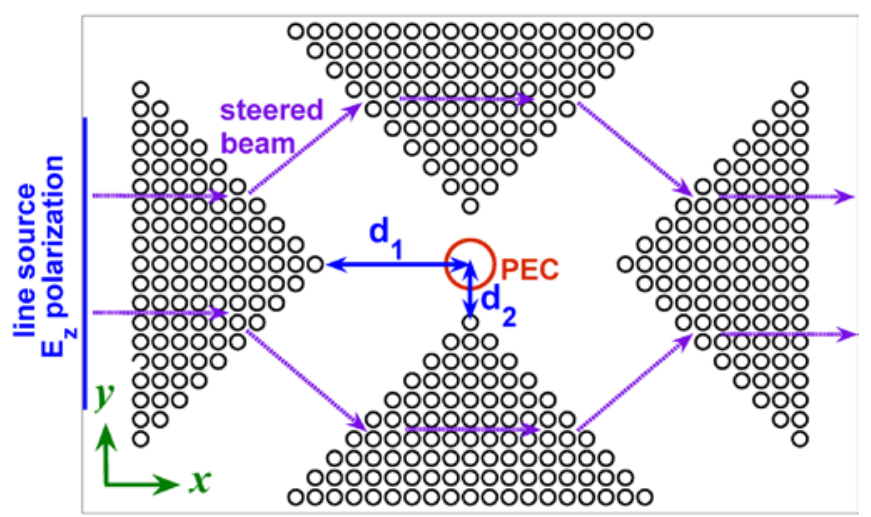

Figure 3: Di rectional c loak ba sed on the be am $\mathrm{s}$ teering effect at higher photonic bands. $\mathrm{d}_{1}$ and $\mathrm{d}_{2}$ are the horizontal and vertical separation of the prism wedges respectively.

\subsection{Wavefront Reconstruction}

Field $\mathrm{c}$ omputations are $\mathrm{p}$ erformed $\mathrm{f}$ or $\mathrm{t}$ he $\mathrm{p}$ roposed geometry shown in Figure 3 using the e-m solver COMSOL RF Module [13]. A line source of $12 \mathrm{~cm}$ with the TM mode polarization is used for the normal incident excitation. The geometry is surrounded by the computational domain with a size of $32 \mathrm{~cm} \times 20.5 \mathrm{~cm}$ and the open space is terminated by the absorbing bo undary conditions. $\mathrm{S}$ imulations are do ne for the in-plane system, where the height of the PC rods is assumed to be infinite.

Figure $4 \mathrm{~s}$ hows th e $\mathrm{E}_{\mathrm{z}}$ field $\mathrm{m}$ ap at $26.77 \mathrm{G} \mathrm{Hz}$ for a normal incident e-m wave impinged on the $\mathrm{PC}$ geometry for three different cases namely; (a) without PEC, (b) with PEC and (c) a ir b ackground respectively. $I t$ is $f$ ound $t$ hat $t$ he beam steering reconstructs the e-m beam at the output port and $\mathrm{t}$ heir wavefront $\mathrm{s}$ hape i s s imilar $\mathrm{t}$ o $\mathrm{t}$ hat $\mathrm{o} \mathrm{ft}$ he a ir background. This is further revealed in the field scanning plot in Figure 4(d), which shows the scanned field profiles for al $1 \mathrm{t}$ hree cases at $26.77 \mathrm{G} \mathrm{Hz}$. Though $\mathrm{t}$ he $\mathrm{r}$ ecorded profiles are influenced by the scattering losses, the observed feature is a desired as pect for the c oncealment $u$ tility i $n$ microwave communication systems.

It is learnt that the prism element with the effective index less $\mathrm{t}$ han $\mathrm{t}$ he $\mathrm{a}$ ir $\mathrm{m}$ edium e ntails $\mathrm{t}$ he realization of a directional $\mathrm{c}$ loak a $\mathrm{t} \mathrm{l}$ east $\mathrm{f}$ or a $\mathrm{n}$ ormal i ncident, 1 inearly polarized e $-\mathrm{m} \mathrm{w}$ aves. It i s i nteresting t o $\mathrm{n}$ ote $\mathrm{t}$ hat $\mathrm{s}$ uch approach $\mathrm{c}$ an also be attempted with the other mesoscopic systems such as the quasi-periodic, non-periodic and indefinite media. Hence, it is a nticipated that optimization of $t$ he $p$ roposed di rectional $c$ loak with respect $t$ o $t$ he scattering losses, polarization state, incident angle will lead to the practical utilities in radar and communication applications.

\section{Conclusions}

Beam s teering a rising from the hi gher photonic ba nds in dielectric $\mathrm{p}$ hotonic $\mathrm{c}$ rystal i s $\mathrm{r}$ eported i $\mathrm{n} \mathrm{t}$ his work. I $\mathrm{t}$ i s found $t$ he investigated dispersion $r$ egimes possess $t$ he effective index less than the air background. This steers the normal incident beam in a positive direction in $\mathrm{PC}$ wedge. This property allows one to design the beam expanders and compressors based on the prism combinations.
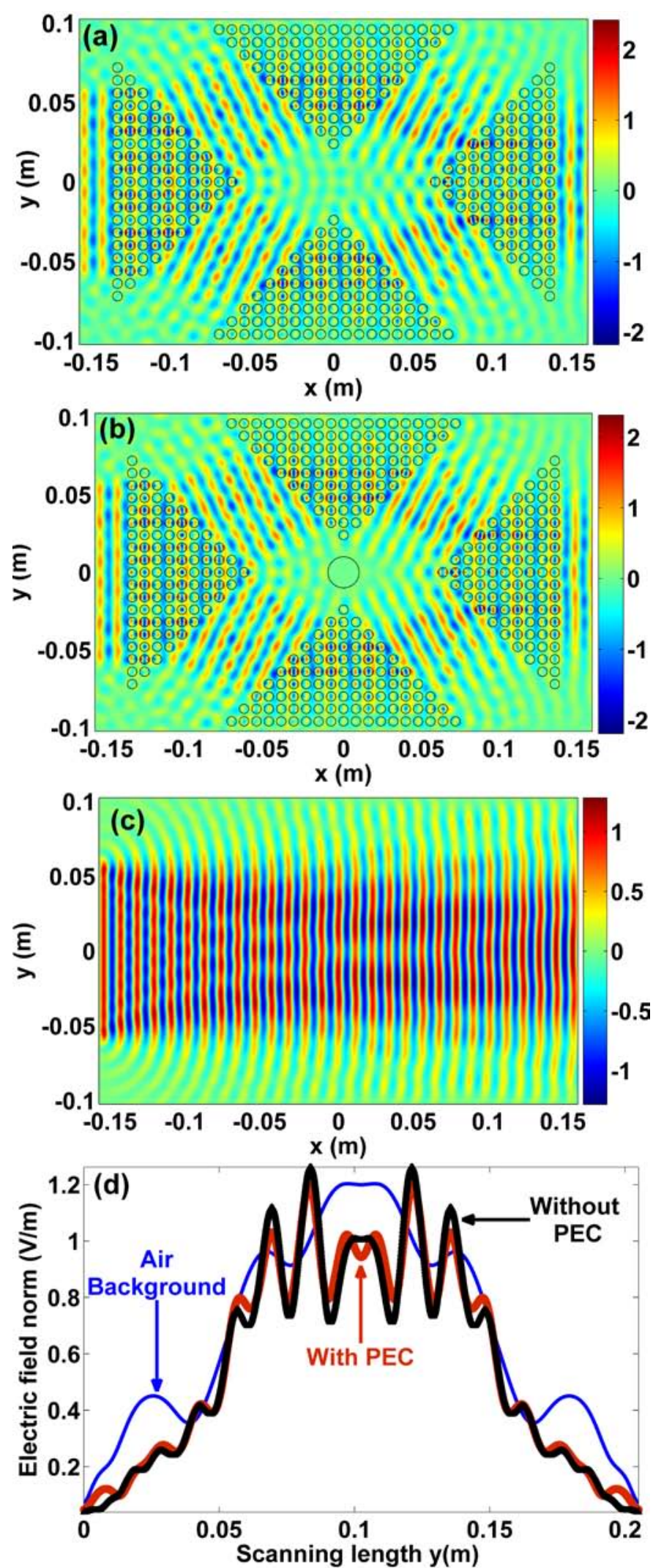

Figure $4 \mathrm{E}_{\mathrm{z}}$ field maps at $26.77 \mathrm{GHz}$ for (a) empty (b) PEC loaded PC geometry a nd (c) a ir background $r$ espectively. (d) S canned el ectric field profiles al ong the $y$ direction at the detecting plane at $x=0.157 \mathrm{~m}$. 
As a $\mathrm{n}$ a pplication point of $\mathrm{v}$ iew, steering $\mathrm{c}$ oncept i s employed for t he de sign of a di rectional c loak. It i $\mathrm{s}$ numerically demonstrated that the pr oposed de sign effectively reconstructs the e $-\mathrm{m}$ b eam and it maintains the wave s hape s imilar t o the a ir background. This a spect is useful for the $\mathrm{d}$ evelopment o f $\mathrm{c}$ oncealment utilities a nd stealth t echnology i $\mathrm{n} \mathrm{m}$ icrowave systems. O ptimizing the design with respect $t$ ot he $\mathrm{s}$ cattering losses, oblique incidence a nd p olarization state $\mathrm{w}$ ill e ntail t he real t ime utilities of a directional cloak.

\section{References}

[1] J.D. J oannopoulos, S .G. J ohnson, J .N. Winn, R .D. Meade, Photonic crystals molding the flow of light, Princeton University Press, Princeton, Ch-10, pp. 190 228, 2008.

[2] Y.A. U rzhumov, D.R. Smith, T ransformation o ptics with pho tonic ba nd g ap m edia, Phys. Rev. Lett 105: 163901, 2010.

[3] O. V anbésien, N . Fabre, X. M élique, D. L ippens, Photonic $\mathrm{cr}$ ystal b ased $\mathrm{cl}$ oaking d evices at o ptical wavelengths, Appl. Opt 47: 1358-1362, 2008.

[4] B. Va sić, R. Gajić, Self-focusing m edia u sing graded photonic c rystals: F ocusing, F ourier tr ansforming a nd imaging, directive emission, and directional cloaking, J. Appl. Phys 110: 053103, 2011.

[5] N. Yogesh, V. S ubramanian, D irectional cl oak formed by photonic crystal waveguides, Microwave symposium digest (2012), IEEE MTT-S International. Montreal, Canada, pp. 1-3, 2012.

[6] N. Y ogesh, V . Subramanian, S ource transformation device formed by o ne-dimensional $\mathrm{p}$ hotonic c rystals, Opt. Lett 36: 1737-1739, 2011.

[7] D. S churig, J.J. Mock, B.J. Justice, S.A. Cummer, J.B. Pendry, A .F.S tarr, D.R.S mith, $M$ etamaterial electromagnetic cl oak at microwave $\mathrm{f}$ requencies, Science 314: 977-980, 2006.

[8] T. Han, X. T ang, E xternal cloak w ith h omogeneous material, J. Phys. D: App. Phys 42: 235403, 2009.

[9] T. Ha n, C .-W. Q, I sotropic n onmagnetic f lat c loaks degenerated from homogeneous anisotropic trapeziform cloaks, Opt. Exp 18: 13038-13043, 2010.

[10] J. Zhang, L. Liu, Y. Luo, S. Zhang, N. A. Mortenson, Homogeneous o ptical c loak c onstructed with uniform layered structures, Opt. Exp 19: 8625-8631, 2011.

[11] N. Landy, D. R. Smith, A full-parameter unidirectional metamaterial cloak for microwaves, Nat. Mat 12: 25-28, 2013.

[12] J.P. Turpin, A .T. Massoud, Z .H. Jiang, P .L. W erner, D.H. We rner, $C$ onformal mappings to achieve s imple material $\mathrm{p}$ arameters for $\mathrm{t}$ ransformation o ptics d evices, Opt. Exp 18: 244-252, 2010.
[13] J. R. Whiteman, The mathematics of finite elements and applications, John Wiley a nd S ons, C hichester, 1998, Version COMSOL 4.2, http://www.comsol.com

[14] S.G. J ohnson, J .D. J oannopoulos, B lock-iterative frequency-domain methods for Maxwell's equations in a plane wave basis, Opt. Exp 8: 173-190, 2001. http://abinitio.mit.edu/mpb 